\title{
Cognitive brain potentials in kindergarten children with subtyped risks of reading retardation
}

\author{
Dirk J. Bakker • Jan W. Van Strien • Robert Licht • \\ Sietsia W. D. Smit-Glaudé
}

Received: 6 June 2006 / Accepted: 4 February 2007 / Published online: 24 May 2007

(C) The International Dyslexia Association 2007

\begin{abstract}
Cognition-related brain responses to meaningful and meaningless figures were registered in 5-year-old kindergarten children who either had been subtyped as being at-risk of developing an L- or P-type dyslexia (LAL versus LAP) or who were not at-risk. While identifying, naming, or categorizing pictures, event-related potentials (ERP) were registered. Three cognition-related components were found: the N460, the P780, and the Slow Wave (SW). LAP-children produced weak N460 activity across tasks, whereas LAL children, and to a lesser degree, non-risk children produced robust task-dependent activity. This finding may indicate that LAP-children lack semantic input while processing the figures. P780 latencies to frequently occurring figures were found hemisphere-dependent: LAP-children showed longer latencies in the right than in the left hemisphere, whereas the distribution was reversed in the LAL and non-risk children. It was also found that the right hemisphere is generally responsible for a lion's share of the processing of figures and therefore it seems that the right hemisphere of LAP-children invests ample time in doing so. Whereas LAP-children showed largest SW amplitude differences between frequent and infrequent stimuli at posterior locations, LAL children did so at frontal locations. Assuming
\end{abstract}

This manuscript was peer reviewed, processed and accepted under the editorship of the immediate past editor Dr. Che Kan Leong.

D. J. Bakker · R. Licht

Vrije Universiteit, Amsterdam, The Netherlands

R. Licht

e-mail: r.licht@psy.vu.nl

J. W. Van Strien

Erasmus Universiteit, Rotterdam, The Netherlands

e-mail: vanstrien@fsw.eur.nl

S. W. D. Smit-Glaudé

Sociaal Medisch Centrum Snouck van Loosen,

Enkhuizen, The Netherlands

e-mail: info@dyslexie.nu

D. J. Bakker $(\bowtie)$

Beerstratenlaan 47, 2421 GN Nieuwkoop, The Netherlands e-mail: dirkjbak@planet.nl 
that the SW represents working-memory processes, it may be that working-memory in LAPchildren deals with figure-relevant visual-spatial information and with figure-derived concepts in LAL children. Overall, the findings suggest that LAL and LAP represent two different groups of kindergartners at risk of dyslexia and that these differences, to some degree, fit with the presumed etiology of L- and P-type dyslexia.

Keywords Kindergarten children · Children at-risk of reading problems · ERP-validation of subtyped risks

\section{Introduction}

According to Bakker and associates (Bakker, 1979, 1990, 2002, 2006; Licht, Bakker, Kok, \& Bouma, 1988; Robertson, 2000), the initial and advanced phase in the learning to read process are different in nature. The novice reader is faced with letter shapes which, unlike the shapes of familiar objects, are new for him or her. Letters are different from common shapes in a number of respects. First, letters do not show shape constancy, which is to say that the same letter shapes in different spatial positions may represent different meanings. For instance, a "d", "p", "b", and a "q", while having the same shape are different letters. On the other hand, an apple is an apple whether seen from above or from below. Second, whereas the meaning of a common shape changes when the shape changes, changing " $\mathrm{d}$ " to " $\mathrm{D}$ " does not change the meaning. Third, different groupings of the same letters may produce different words: "name", "mane", "mean", "amen". A railway-train though remains a railway-train, regardless of changes in the sequence of coaches. Finally, changing the sequence of words within a sentence may completely change the meaning of that sentence: Compare, "the cow is on the car" with "the car is on the cow". Thus, mastering script must be quite a perceptual burden for a novice in the primary school. Due to the requirement of perceptual script analyses, early reading will predominantly be mediated by the right cerebral hemisphere.

At the time the child gets more experienced with script, his reading strategies will change accordingly. First, perceptual analyses became an automatic process. Second, the lexicon became larger and richer over the years. Third, the ultimate aim of reading not only is accuracy but also fluency, the latter not being compatible with painstakingly analyzing the perceptual features of text. In view of these changes, a more linguistic reading strategy is called for. Based on a rich lexicon and mastered syntactic rules, the fluent reader is able to quickly test the meaning of a sentence, a process that does not require to address every word of that sentence. Advanced reading, while relying on lingual strategies, thus alludes to predominant left hemispheric processing. Licht et al. (1988), in a longitudinal study spanning the period kindergarten through grade three of the primary school, found electrophysiological evidence for the hemispheric shift in the predominant mediation of reading.

A child may get stuck in the analysis of the surface structure of text, that is, in the generation of right hemispheric reading strategies. Such a child remains in the initial phase of learning to read, and thus, will read slowly but relatively accurately. This style of reading failure has been denoted P-type dyslexia; these readers are also called "spellers" (Van der Schoot, Licht, Horsley, \& Sergeant, 2000). Other readers may start to generate left hemispheric, linguistic strategies too early. As a result, they will read relatively fast, but as they tend to overlook the perceptual properties of text, they will produce many errors. These readers were denoted L-type dyslexic children or "guessers" (Van der Schoot et al., 2000). A number of investigators mention a third type, a mixed or M-type of developmental dyslexia (e.g., Fabbro, Pesenti, Facoetti, Bonanomi, Libera, \& Lorusso, 2001). M-type dyslexic children read slowly and inaccurately. 
The studies discussed concern subtypes of manifest dyslexia, the present study though concerns the validity of sub-typed reading risks (LAL versus LAP) in pre-reading kindergarten children. In a companion study (Smit-Glaudé, Van Strien, Licht, \& Bakker, 2005), it was argued that a visual-perceptual versus a verbal bias in the processing of cognitive information by children at-risk may be precursory of manifest P- and L-dyslexia, respectively. First year kindergartners, considered to be at-risk by their teachers for developing reading difficulties in the Smit-Glaudé et al. study, were therefore presented a screening test to investigate a possible visual-perceptual versus verbal bias in their performance. These biases were found indeed.

The present investigation aims to find cognition-related, electrophysiological reflections of the verbal versus visual-spatial processing bias in pre-reading LAL versus LAP children, respectively. Electrophysiological research, in relation to language and reading performance in childhood, progresses quickly. Exemplary are investigations by Molfese and associates (for an overview see Molfese, Molfese, Key, Modglin, Kelley, \& Terrell, 2002) and by the group of Lyytinen (e.g., Lyytinen, Leppänen, Richardson, \& Guttorm, 2003). Most of these studies are longitudinal in nature and aim to relate language and reading performance in school children with brain responses to speech and nonspeech stimuli presented at the time these children were neonates and toddlers. Some of the outcomes reported by Molfese et al. are of special interest in the context of the present research in that at least three of the six components of the brain responses recorded at neonatal age - which were found to predict later reading performanceappeared to be located in the right hemisphere. In the present investigation, we predict that the verbal versus visual-spatial processing bias in kindergartners with subtyped risks of dyslexia are hemisphere-tied. In other words, we predict LAL versus LAP to interact with the left versus the right hemisphere in the way these children process the cognitive information presented.

Indeed, a verbal versus visual-spatial bias in the processing of cognitive information, and by inference, a predominant left versus right hemispheric subservience in the processing of script and script-like information, was and still is thought to be fundamental in the etiology of L- and P-dyslexia (see Bakker, 2002, 2006). Recently though, a number of studies (Lorusso, 2006) have investigated the possible mechanisms, hemisphere-tied or otherwise, which correlate with or even underlie the L/P-distinction. Attention, inhibition especially, has been shown to be of primary importance in the differentiation of the dyslexia subtypes. Van der Schoot et al. (2000) demonstrated that L-types have more difficulty in inhibiting an ongoing response than P-types. A subsequent investigation (Van der Schoot et al., 2002) showed this inhibition difficulty to be related to a fronto-central processing disturbance in L-dyslexic but not so in P-dyslexic children. Van Strien (1999) showed Land P-dyslexic children to produce different learning and short-term memory curves, indicating that memory may be another type-differentiating mechanism. That working memory and/or short term memory plays such a role is supported by the findings of Fabbro et al. (2001) who had L-, P-, and M-type dyslexic children touched fingers of the one hand to be identified on the other hand, a task that requires trans-callosal transfer of memorystored information. L- and M-types were found significantly worse in this task than P-types. Van Strien, Bakker, Bouma, and Koops (1990) found that a number of cognitive processes not only differentiates between $\mathrm{L}$ and $\mathrm{P}$ dyslexic children but also between their biological parents, their fathers especially.

Based on the claim that the cognitive processing differences between L- and P-dyslexia are hemisphere-tied, the hypothesis to be investigated is that LAL and LAP type risk children show differences in the hemispheric distribution of cognition-related brain potentials as, in the present case, elicited by script-substituting stimuli. In view of recent findings concerning type-differentiating mechanisms - as revealed by attention, memory, and other cognitive tasks - it seems worthwhile to explore the elicited potentials in relation 
to these mechanisms. The information gathered may induce suggestions for the refinement, or even the correction, of the balance model of dyslexia.

\section{Method}

Participants, tests and subtyping

In Smit-Glaudé et al. (2005) a full account of subject selection, test administration, outcome analysis, and subtype classification is presented. Therefore, only an overview is given here.

The teachers of an entire school district (135 schools; 1271 children) were requested to indicate whether a child would develop a future reading problem most likely, likely, doubtfully, unlikely, or most unlikely. The number of children considered most likely or likely at risk was 193. After removal of children who, for various reasons, were not further available for the study, a total of 141 children (mean age 5.1 years; 42 girls, 99 boys) were presented the Dutch version of the Florida Kindergarten Screening Battery (Dekker \& Van der Vlugt, 1982). The subtests administered were:

Peabody Picture Vocabulary Test The test taps passive word knowledge (L. Dunn \& L. Dunn, 1981; Manschot \& Bonnema, 1974). The subject (S) indicates which one of four pictures fits a word spoken by the tester (T).

Word Knowledge The test taps active word knowledge (Dekker \& Van der Vlugt, 1982). S is required to mention as many words as possible, within each of the categories: "What can you eat?", "What can you play with?", "What can you see in school?", and "Which animals can you mention?".

Auditory Analysis The test measures auditory analysis of words (Beery \& Buktenica, 1967; extended version). $\mathrm{S}$ is required to repeat words spoken by $\mathrm{T}$ and to do this once again, but this time without a part of the word spoken by T. Degree of difficulty increases gradually, for instance "teacup" without "tea" and later on "cup" without "p".

Developmental Test of Visual-Motor Integration The test measures the integration of visual and motor performance (Beery \& Buktenica, 1967; extended version). S is required to copy 24 geometric figures, one by one, while the target figure is visible all the time. The complexity of the figures increases gradually.

Recognition-Discrimination The test measures visual discrimination performance (Small, 1969). $\mathrm{S}$ is required to indicate which one of four figures is exactly the same as the target figure at the top of the page. The four figures can be rotated or mirrored or can be different in a detail.

Rapid Naming The test measures the automatized naming of pictures (Dekker \& Van der Vlugt, 1982). The test consists of rows of common objects, in different order, which $\mathrm{S}$ is required to name, from left to right, and to do so as fast as possible.

Alphabet Reciting and Counting The test measures the incidental learning of series (Dekker \& Van der Vlugt, 1982). S is required to say the alphabet, and thereafter, to count for one and-a-half minute, starting with the digit one.

The outcomes were compared with those of a random group of kindergartners of comparable age, sampled in an area outside the area of the at-risk children. These control children performed 
significantly better on virtually all subtests. Smit-Glaudé (2003) showed that many children atrisk, when they arrived in the primary school at the end of the first grade, did not master the reading readiness skills that normally are mastered after a few months of reading instruction. Letter naming, phoneme blending, and the visual blending of two subsequent consonants with the remaining of a meaningful word, appeared to be an impossible task for respectively 35 , 41 , and $62 \%$ of these children (across subtypes). This being the case it can hardly be a surprise that Smit-Glaudé et al. (2005), at the end of first grade primary school, found months of backwardness in both word and sentence reading.

The results of the at-risk children were analyzed through a principal components analysis (PCA). Two components emerged, a visual-spatial component and a verbal component. Classification into latent L-types (LAL group) and latent P-types (LAP-group) was based on the outcomes of two discriminant analyses (DA). In the one DA, the raw scores were used in the subtests that loaded highly on the visual-spatial component that emerged from the PCA: Recognition Discrimination and Beery Visual-Motor. In the other DA, the raw scores of tests that loaded highly on the verbal factor were entered: Peabody and Word Knowledge. The number of children that could be classified as LAP or LAL was supplemented with children whose partial component scores in the PCA either revealed greater visual-spatial than verbal values (LAP) or vice versa (LAL). In total, 41 LAP- and 45 LAL children could be classified.

\section{Event-related potentials}

As the present LAL- and LAP children, and those in the control group, were not yet able to read, it was decided to present them a figure-naming task. The ERP-investigation happened about 4 months after the administration of the Florida Kindergarten Screening Battery. The parents of 41 children (mean age: 5.3 years; age range: 4.9-6.5 years) agreed to have their children take part. Of these, 18 were LAL-type risk children (15 boys, 3 girls), 16 were LAP-type risk children ( 8 boys, 8 girls), and 7 were non-risk children ( 3 boys, 4 girls). The latter were drawn from the pool of children who according to the teachers were not likely to develop difficulty in reading. For various reasons though (too much tension, not understanding the instruction, too many eye movements), the data of only 30 children could be used (LAL: $n=13,11$ boys and 2 girls; LAP: $n=10,3$ boys and 7 girls; control group: $n=7,3$ boys and 4 girls).

ERP-recording took place in the psychophysiology laboratory of the university. EEG activity was recorded from 12 tin electrodes, positioned according the 10-20 system at left and right frontal (F3-F4), central (C3-C4), anterior temporal (T3-T4), posterior temporal (T5-T6), parietal (P3-P4), and occipital (O1-O2) sites. These electrodes were embedded in an elastic cap (ECI electro-cap), with FPz serving as ground electrode. The EEG was referenced to the linked earlobes and to control for eye movements, electrodes were placed above and below the right eye and lateral to both eyes. Electrode resistances were kept below $10 \mathrm{kOhm}$. The signals were sampled with a frequency of $100 \mathrm{~Hz}$ and were amplified with a bandpass of .013 to $35 \mathrm{~Hz}$. ERP epochs were extracted with a 1,450-ms duration, beginning $250 \mathrm{~ms}$ before stimulus onset. EEG changes caused by eye movements were corrected by regression and artifacts (more than 150microV difference in the same epoch) were removed. The children were in a sound-proof and electricity-shielded room, lying on a bed, with their head on a vacuum pillow. The order of presentation of three ERP subtasks was counterbalanced across the participating children.

\section{ERP-tasks}

Figure-naming seems to be a good substitute for word-naming as the naming of figures appeals to the same hemispheric processes as does the naming of words (Dencla \& Rudel, 
1974; Jansky \& De Hirsch, 1972). The figure-naming task came in three parts: a figurenaming task, a control task, and a figure-categorization task. Within each task, frequent and infrequent stimuli were presented. This was done since Stuss, Picton, and Cerri (1986) found the N400 peak to become more negative with an increasing number of different infrequent stimuli, which may be due to an increasing memory load.

In the first subtask meaningful figures, replacing a fixation circle in the middle of a screen, had to be named aloud. The stimuli were a house, a fish, a cap, a cat, and a boat. In $50 \%$ of the trials, the figure of a house was presented (frequent stimulus), and in 50\%, four different figures were presented, each of these five times (infrequent stimuli), making 40 trials in total. The child had to respond upon hearing a tone that was given after the presentation of a figure. As a control on the first subtask, a second subtask was presented, requiring the child to say "yes" to the appearance of a nonsense figure (random line drawings). The nonsense figures, while appearing at the center of a screen, had to be responded to upon hearing a tone that came after stimulus presentation. In $50 \%$ of the trials, the same nonsense figure was presented (frequent stimulus), and in $50 \%$ four different figures, each were presented five times (infrequent stimuli), making 40 trials in total. The third subtask was the same as the first subtask except that now the child had to say whether the figure was a house or not (category decision). The three subtasks were presented in random order. All figures measured $2 \times 3 \mathrm{~cm}$ and came in yellow to a dark background. The child was seated at $70 \mathrm{~cm}$ in front of the screen. The physical characteristics, especially contrast and light intensity, were kept equal across figures. In each trial, a figure was presented for $2,000 \mathrm{~ms}$. To prevent a contingent negative wave, it was decided to vary the time interval between the appearance of the fixation circle and the onset of a figure: 1,100,1,400, or $1,700 \mathrm{~ms}$. The intertrial time varied from 400 to $1,600 \mathrm{~ms}$. Before testing, the child practiced with the tasks until complete understanding was accomplished.

\section{Data analysis}

The ERP-signals were defined relative to the 250 -ms pre-stimulus baseline period and were averaged per child and condition. To separate the 1,200 ms waveform into appropriate components, a temporal principal component analysis (PCA) of the ERP data was conducted. After condensing the time domain with a factor 2 (resulting in 60 data points per 1,200 ms epoch), the PCA was carried out on the covariance matrix of the 216 waveforms of the present experiment ( 3 groups $\times 12$ electrodes $\times 6$ conditions). The number of factors was limited to the number of eigenvalues greater than one. Five factors were extracted and rotated to the Varimax criterion. The results of the PCA are summarized in Table 1. The rotated factor loadings of the five PCA-factors plotted against the 1,200-ms sampling epoch are shown in Fig. 1.

Three of these factors concern cognition-related, endogenous components: the N460, peaking between 300 and $700 \mathrm{~ms}$; the P780, peaking between 750 and $800 \mathrm{~ms}$; the Slow Wave (SW, 800-1200 ms), which together account for $85 \%$ of total variance. The exogenous factors $\mathrm{N} 180$ and $\mathrm{P} 300$ account for $5 \%$ of total variance. In the present study, we

Table 1 Results of the PCA on the ERPs elicited by figures

\begin{tabular}{clcc}
\hline Factor & Component & Percent variance (\%) & Percent cumulative (\%) \\
\hline 1 & N460 & 62 & 62 \\
2 & SW & 17 & 79 \\
3 & P780 & 6 & 85 \\
4 & P300 & 3 & 88 \\
5 & N180 & 2 & 90 \\
\hline
\end{tabular}


Fig. 1 PCA rotated factor loadings for N180, P300, N460, P780, and SW

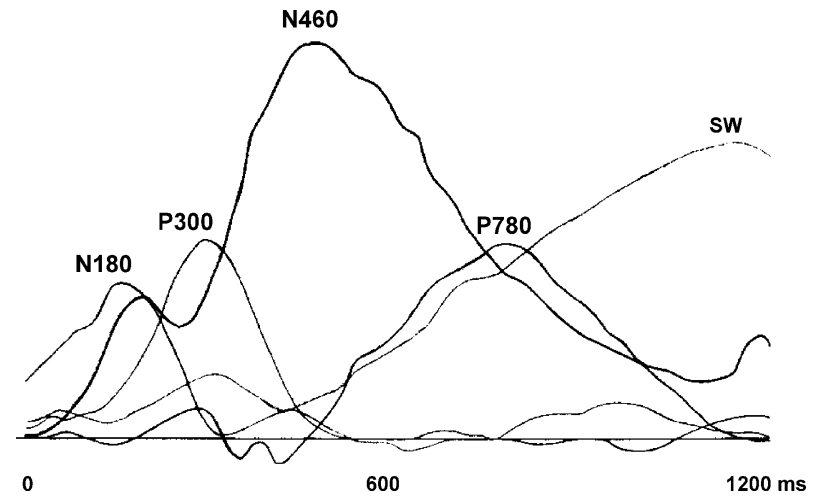

were interested in cognition-related processing only. As endogenous, rather than exogenous components reflect cognitive processing, only the endogenous components were analyzed.

Along with the PCA-factors, mean amplitudes were established within the time zones that showed the highest PCA-factor loadings, for the N460, P780, and SW. The latencies of the various peak amplitudes were also established (F3/4, C3/4, T3/4, P3/4). Data were analyzed through analyses of variance (ANOVAs), with Group as between-subjects factor and Condition (three tasks), Frequency (frequent/infrequent stimuli), Hemisphere (left/ right), and Location (various electrode positions) as within-subjects factors. When applicable, $F$-ratios were tested with Greenhouse-Geisser corrected degrees of freedom.

\section{Results}

Hemisphere-tied group effects

As the balance model of learning to read and dyslexia is based on the phase-dependent involvement of the right and left cerebral hemisphere in reading, lateral differences in the processing of figures by LAL and LAP children were predicted. Hemispheric differences showed up for P780 latencies only: Group $\times$ Hemisphere $[F(2,27)=7.75 ; p<0.005]$ and Group $\times$ Hemisphere $\times$ Frequency $[F(2,27)=4.86 ; p<0.02$; Fig. 2] appeared to be significant. These findings indicate that LAP children show longer latencies to frequent stimuli in the right than in the left hemisphere and that LAL and Control children, for frequent stimuli especially, show the reverse pattern. The hemispheric distribution of latencies to infrequent stimuli is about the same across groups.

Fig. 2 Mean P780 latencies as a function of group, hemisphere, and frequency

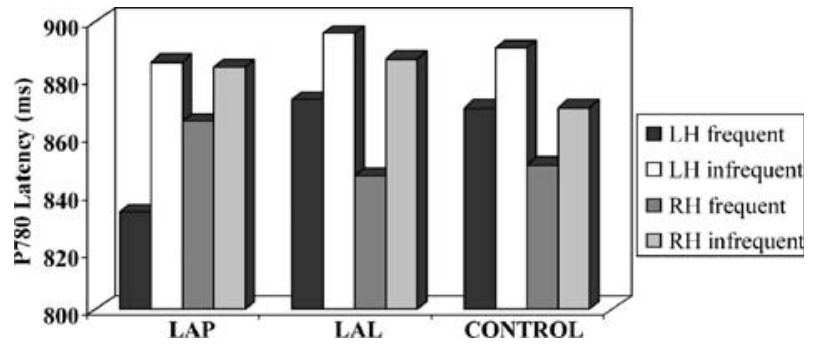

Springer 
Fig. 3 Mean N460 amplitudes as a function of group and condition (vertical axis refers to microvolts; negativity is up)

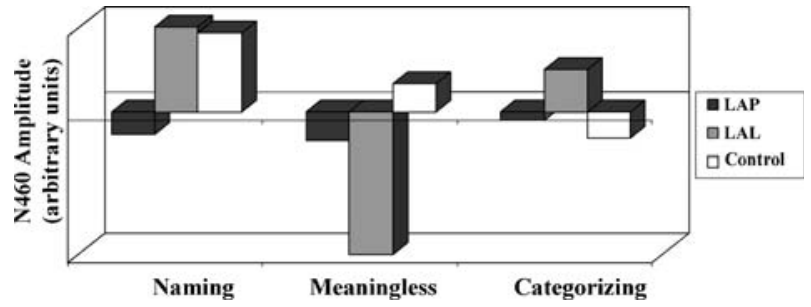

Hemisphere-indifferent group effects

As this research concerns an effort to validate and to amend the LAL/LAP classification, only main and interaction effects of group are presented. Other effects will be discussed if useful for the interpretation of the group effects. The effects concern both amplitudes and latencies.

N460 Group $\times$ Condition (Fig. 3) was significant, both for the PCA-component scores $[F$ $(4,54)=4.42, p<0.005]$ and the mean amplitude $[F(4,54)=5.04, p<0.002]$. LAP-children showed weak N460-activity across conditions. Condition did affect activity in LAL children, and to a lesser degree, in non-risk-children. LAL children demonstrated robust N460 positivity on the control task (meaningless figures) and negativity on the naming and categorization task.

P780 With regard to latencies, Group $\times$ Condition $\times$ Frequency $[F(4,54)=2.98 ; p<0.05]$ was found to be significant. This finding indicates that LAL children show longer latencies to infrequent stimuli in the categorization task than in the control task and that the other two groups (LAP and Control) fail to show this pattern. With regard to frequent stimuli, LAL children showed longer latencies in the control than in the categorization task, whereas the reversed pattern was evident for the LAP children.

$S W$ Group $\times$ Frequency $\times$ Topography was significant, both for the PCA component scores $[F(10,135=3.11 ; p<0.002]$ and mean amplitude $[F(10,135)=2.81 ; p<0.005]$. Control children showed larger SW amplitudes to infrequent than to frequent stimuli at all locations and whereas LAP children showed the largest differences between frequent and infrequent

Table 2 Significant ERP parameters by LAL versus LAP

\begin{tabular}{|c|c|c|}
\hline Parameters & LAL & LAP \\
\hline $\begin{array}{l}\text { N460 } \\
\text { amplitude }\end{array}$ & Condition-dependent strong activity & Condition-independent weak activity \\
\hline \multirow[t]{3}{*}{$\begin{array}{l}\text { P780 } \\
\text { latency }\end{array}$} & $\begin{array}{l}\text { Longer in left than right hemisphere for } \\
\text { frequent stimuli }\end{array}$ & $\begin{array}{l}\text { Longer in right than left hemisphere for } \\
\text { frequent stimuli }\end{array}$ \\
\hline & $\begin{array}{l}\text { Longer in categorization than in control task } \\
\text { for infrequent stimuli }\end{array}$ & $\begin{array}{l}\text { Not longer in categorization than in control } \\
\text { task for infrequent stimuli }\end{array}$ \\
\hline & $\begin{array}{l}\text { Longer in control than in categorization task } \\
\text { for frequent stimuli }\end{array}$ & $\begin{array}{l}\text { Longer in categorization than in control task } \\
\text { for frequent stimuli }\end{array}$ \\
\hline $\begin{array}{l}\text { SW } \\
\text { amplitude }\end{array}$ & $\begin{array}{l}\text { Largest difference in amplitudes between } \\
\text { frequent and infrequent stimuli at frontal } \\
\text { locations }\end{array}$ & $\begin{array}{l}\text { Largest difference in amplitudes between } \\
\text { frequent and infrequent stimuli at posterior } \\
\text { locations }\end{array}$ \\
\hline
\end{tabular}


stimuli at parietal and occipital locations, LAL children showed largest differences at frontal locations.

The effects of LAL versus LAP on the N460, the P780, and the SW amplitudes and/or latencies are shown in Table 2.

\section{Discussion}

The balance model of dyslexia holds that L- and P-typed dyslexic children are differentially biased in the generation left versus right hemispheric reading strategies, and that in consequence, type-specific reading styles emerge. In searching for possible precursors of subtyped dyslexia, it thus seems evident that hemispheric differences should show up in the way pre-reading children with subtyped risks of dyslexia process figures, used as word-substituting stimuli. These hemispheric differences may concern ERP amplitudes, ERP latencies, or both. In the present research, only one significant hemisphere-specific result emerged: the latencies of the P780 to frequently occurring figures, were longer in the right than in the left hemisphere of LAP children and vice versa in LAL and non-risk children. The late positive component P780 can be conceived as a member of the P300 family (Friedman, Brown, Sutton, \& Putman, 1982) and may represent the categorization of a figure and response selection. Identifying, naming, and categorization of figures, of course, is matter for both hemispheres, albeit that the share of the one hemisphere in a particular part of the process can be larger than the share of the other hemisphere. The P780 amplitudes in all groups were found larger over the right than over the left hemisphere [PCA: $F(1,27)=16.52, p<0.001$; Amplitude: $F(1,27)=17.47, p<$ $0.001]$. Thus, the right hemisphere generally stands out at this point in the processing of figures. But the right hemisphere in LAP children seems to invest more time in doing that part of the job, i.e., in concluding that the figure out there represents a house, another object, or something meaningless. If so, one might speculate that from the onset of learning to read, the right hemisphere of a LAP child may invest relatively much time before concluding that for instance, the A-shaped figure, out there on the blackboard, is an "A". That P-type dyslexic children read slowly but relatively accurately would then also be explainable.

The differential hemispheric speed in the processing of cognitive information by LAL and LAP children, as revealed by the P780 latencies, stands alone. This does not mean though that the validity of the LAL versus LAP typing of children at risk of dyslexia lacks further support. As mentioned already, the balance model of learning to read and dyslexia originates from the idea that visual-spatial and lingual strategies predominate in early and advanced reading, respectively, and that the same holds true for P- versus L-type of dyslexic reading. That presumably being the case, it was subsequently assumed that P-type and L-type dyslexic reading is primarily mediated by the right and left hemispheres, respectively. That was why hemispheric differences were predicted in the cerebral manifestations of LAL- versus LAP-type dyslexia. But this prediction leaves unhindered the possibility that perceptual versus lingual differences between LAL and LAP children do emerge and that these strategic differences, for whatever reason are not (yet) hemispheretied. Thus, hemisphere-indifferent LAL versus LAP differences showing up anywhere during the processing of figures, may quite well fit into the balance model of dyslexia.

According to Sitnikova, West, Kuperberg, and Holcomb (2006), the N460 may represent the first stage of semantic processing, i.e., the recognition and naming of the stimulus, 
based on semantic knowledge. The LAP children show attenuated N460 amplitudes to both meaningful and meaningless stimuli, that is, low and stimulus-indifferent activity. One is inclined to wonder if there is much input of semantic knowledge at all in these LAPchildren. Whereas they do not appear to have a tendency towards choosing between meaningful and meaningless, LAL children do have as they show N460 negativity and N460 positivity to meaningful and meaningless figures, respectively. Would it be possible that the near absence of semantic input in this phase of information processing is precursory to the proposed difficulty P-dyslexic children have in switching to a predominant lingual strategy at some point during the learning to read process? Alternatively, could it be the case that LAL dyslexic children, while showing markedly more N460-variation to meaningful versus meaningless figures than the non-risk control children, are biased to semantic processing to such an extent that later on, they use such lingual strategies as soon as learning to read starts? No answer is available to the question why the hemispheres do not interact with group and condition in the generation of semantic knowledge as reflected in the N460 amplitudes, or it should be the trivial answer that the potentials evoked in LAPchildren are so small that no room is left for demonstrating hemispheric variation.

The Slow Wave may represent verbal working-memory processes, involved in maintaining the delayed vocal response (Ruchkin, Grafman, Cameron, \& Berndt, 2003). In the present study, the amplitudes of the Slow Wave were found affected by a number of factors. Overall, the amplitudes appeared to be larger over the right than over the left hemisphere [PCA: $(F(1,27)=7.71, p<0.01$; Amplitude: $F(1,27)=11.97, p<0.002$ ], possibly indicating that the memory load involved in the figure tasks are more a burden for the right than for the left hemisphere. The degree of this burden though is dependent on the interaction of Group, Frequency, and Topography. Non-risk children showed larger amplitudes for infrequent than for frequent stimuli at all scalp locations. A similar effect was found at occipital and parietal locations in LAP children and at frontal locations in LAL children. Thus, a LAL versus LAP difference goes with an anterior-posterior gradient. That being the case, one wonders what kind of working memory is associated with the processing of figures by LAL and LAP children. First, it seems reasonable to assume that infrequent figures load on working memory more than frequent figures, which would explain the amplitudes to infrequent figures to be larger than those to frequent figures. Second, the posterior location of the SW-amplitudes, elicited by infrequent figures in LAP children, suggests that the working memory in these children deals with figure-relevant visual-spatial information. In LAL children, these figures may have been conceptualized, which is to say that it is not (anymore) the "thing out there" (e.g., the outline of a boat), but rather the concept of that thing (e.g., the concept "boat"). This suggestion comes close to what Kok (1988) called an externally versus internally oriented split that he coupled with SW-activity at posterior and anterior locations, respectively.

If it holds true that the validity of a classification is demonstrated when it can be shown that the classes differ on factors that were not included as criteria for that classification, then it is evident that the L/P classification shows validity. LAL and LAP children at risk of dyslexia were found to differ from each other on some ERP-parameters, and in a number of cases, also from non-risk children. The intrinsic value of a demonstrated validity is strengthened though when the found differences between classes can meaningfully be integrated in the theory or model on which the classification is based. In the present case, that theory is Bakker's balance model of learning to read and dyslexia. The steps in the development of that model were: (1) Initial and advanced reading are different in nature in that initial reading proceeds slowly and advanced reading more fluently; (2) Initial reading is bottom-up in that it starts with the perceptual analysis of text features, whereas advanced 
reading is top-down in that it proceeds by applying learned syntactic rules; (3) Initial and advanced reading are subserved by the predominant generation of right and left hemispheric strategies, respectively; (4) P-type dyslexia arises when a child is not able to switch from predominant perceptual to predominant lingual analysis of text, and L-type dyslexia arises when the child disregards the perceptual features of text and use lingual strategies from the very onset of learning to read; (5) P-dyslexic children presumably were stuck in the generation of right hemispheric reading strategies, whereas L-dyslexic children used left hemispheric reading strategies prematurely; (6) Consequently, P-type dyslexic children, by way of treatment, should receive specific stimulation of the left hemisphere, and L-dyslexic children, stimulation of the right hemisphere; (7) In trying to track the kind of mechanisms involved in the reading strategies employed by P- versus L-dyslexic children, investigators recently found that attention and memory play an as yet unknown role.

The present study investigated the possible precursors of manifest L- versus P-dyslexia, focusing on the fifth step in the development of the balance model, i.e., on hemispheric differences in the processing of figures. One hemispheric difference was found: P780 latencies were longer in the right than in the left hemisphere of LAP children and vice versa in LAL children. This result suggests that the right hemisphere of LAP children invests more time in finding out what a figure represents. In doing so, LAP children, more than LAL children, seemingly want to be convinced that no constituent part of the figure is overlooked. This of course would fit in with the way LAP children process text later in life. Two other results of primary interest emerged, none of these showing ties with predominant left or right hemispheric processing (step 5) but which rather fit in with steps 4 and 7 . In line with the idea that P-type dyslexic children are not prone to employ lingual strategies in the processing of text (step 4), the present LAP children generated low N460 activity, presumably indicating that there is little semantic input in the recognition of figures. Fitting in with step 7, it was found in this research that working memory-as expressed in the SWamplitudes to infrequent stimuli especially_-shows different cortical locations of primary subservience: anterior locations in LAL children, posterior locations in LAP children, findings that call to mind those of Van der Schoot, Licht, Horsley, and Sergeant (2002). It seems that LAL children are inclined to rehearse concepts (internal orientation), and LAP children are inclined rather to rehearse percepts (external orientation). LAP children may have failed to build up an appropriate (semantic) lexicon.

In general, the conclusion seems to suggest that a limited number of outcomes can reasonably be considered to reflect processes that underlie the etiology of L- and P-dyslexia. That only one of these outcomes is hemisphere-tied provides subject matter for thought; the more so because there is now ample evidence that single hemisphere stimulation is effective in the treatment of dyslexia (e.g., Bakker, 2006; Lorusso, Facoetti, Paganoni, Pezzani, \& Moltini, 2006). That being the case, one would expect the biases in the hemispheric subservience of text and text-substituting material to be prominent and easy to disclose.

However that may be, the present study calls for some caution. First, the number of participants in the present ERP-study was limited. Second, as the inclusion of children depended on the permission by the parents, one should not consider the participants in the ERP-study to be a random subgroup of the participants in the companion investigation (Smit-Glaudé et al., 2005). Third, division of gender did not appear to be equal over groups in the present study. Thus, the status of the results is provisional. That being the case, the outcomes by and large are in support of the theory that an early visual-spatial processing bias, primarily subserved by the right cerebral hemisphere versus an early verbalconceptual bias, primarily subserved by the left cerebral hemisphere, are at the roots of two subtypes of latent (LAP versus LAL) and manifest (P-type versus L-type) dyslexia. 
Acknowledgements We would like to thank "Stichting Kinderpostzegels Nederland" (Netherlands' Children's Welfare Stamp Fund) for financially supporting this project and SABD "Gelders Rievierengebied" for allowing us to carry out this work in their school district.

\section{References}

Bakker, D. J. (1979). Hemispheric differences and reading strategies: Two dyslexia's? Bulletin of the Orton Society, 29, 84-100.

Bakker, D. J. (1990). Neuropsychological treatment of dyslexia. New York: Oxford University Press.

Bakker, D. J. (2002). Teaching the brain. In A. Y. Stringer, E. L. Cooley, \& A. L. Christensen (Eds.), Pathways to prominence in Neuropsychology: reflections of twentieth-century pioneers (pp.101-117). New York: Psychology Press.

Bakker, D. J. (2006). Treatment of developmental dyslexia: a review. Pediatric Rehabilitation, 9, 3-13.

Beery, K., \& Buktenica, N. A. (1967). Developmental test of visual-motor integration (extended version). Chicago: Fottlep.

Dekker, J., \& Van der Vlugt, H. (1982). The Florida kindergarten screening battery, Dutch version. Tilburg, Netherlands: Catholic University Brabant.

Dencla, M. B., \& Rudel, R. G. (1974). Rapid 'automatized' naming of pictures, objects, colors, letters and numbers by normal children. Cortex, 10, 186-202.

Dunn, L., \& Dunn, L. (1981). Peabody picture vocabulary test-revisited. Circle Pines, MN: American Guidance Service.

Fabbro, F., Pesenti, S., Facoetti, A., Bonanomi, M., Libera, L., \& Lorusso, M. L. (2001). Callosal transfer in different subtypes of developmental dyslexia. Cortex, 37, 65-73.

Friedman, D., Brown C., Sutton, S., \& Putman, L. (1982). Cognitive potentials in a picture-matching task: comparison of children and adults. In A. Rothenberger (Ed.), Event-related potentials in children (pp. 325-336). Amsterdam: Elsevier.

Jansky, J., \& De Hirsch, K. (1972). Preventing reading failure. New York: Harper and Row Publishers.

Kok, A. (1988). De psychologische betekenis van hersenpotentialen [The psychological significance of brain potentials]. Enschede, Netherlands: Sneldruk.

Licht, R., Bakker, D. J., Kok, A., \& Bouma, A. (1988). The development of lateral event-related potentials (ERPs) to word naming: a four year longitudinal study. Neuropsychologia, 26, 327-340.

Lorusso, M. L. (2006). Neuropsychological factors in the rehabilitation of developmental dyslexia. Unpublished doctoral dissertation, State University, Groningen, Netherlands.

Lorusso, M. L., Facoetti, A., Paganoni, P., Pezzani, M., \& Moltini, H. (2006). Effects of visual hemispherespecific stimulation versus reading-focused training in dyslexic children. Neuropsychological Rehabilitation, 16, 194-212.

Lyytinen, H., Leppänen, P. H. T., Richardson, U., \& Guttorm, T. (2003). Brain functions and speech perception in infants at risk for dyslexia. In V. Csepe (Ed.), Dyslexia: different brain, different behaviour (pp. 113-152). Dordrecht, Netherlands: Kluwer.

Manschot, W., \& Bonnema, J. Th. (1974). Handleiding bij de experimentele Nederlandse normering van de Peabody picture vocabulary test [Manual, Dutch standardization of the PPVT]. Lisse, Netherlands: Swets \& Zeitlinger.

Molfese, D. L., Molfese, V. J., Key, S., Modglin, A., Kelley, S., \& Terrell, S. (2002). Reading and cognitive abilities: longitudinal studies of brain and behavior changes in young children. Annals of Dyslexia, 52, 99-119.

Robertson, J. (2000). Dyslexia and reading: A neuropsychological approach. London: Whurr.

Ruchkin, D. S., Grafman, J., Cameron, K., \& Berndt, R. S. (2003). Working memory retention systems: a state of activated long-term memory. Behavioral and Brain Sciences, 26, 709-777.

Sitnikova, T., West, W. C., Kuperberg, G. R., \& Holcomb, P. J. (2006). The neural organization of semantic memory: electrophysiological activity suggests feature-based segregation. Biological Psychology, 71, $326-340$.

Small, N. (1969). Levels of perceptual functioning in children: A developmental study. Unpublished doctoral dissertation, University of Florida, Gainesville.

Smit-Glaudé, S. W. D. (2003). Een onderzoek naar de voorlopers van P-en L-dyslexie en de effecten van neuropsychologische, preventieve interventie [An investigation into the precursors of $\mathrm{P}$ - and L-dyslexia and the effects of neuropsychological, preventive intervention]. Unpublished doctoral dissertation, Vrije Universiteit, Amsterdam, The Netherlands. 
Smit-Glaudé, W. D., Van Strien, J. W., Licht, R., \& Bakker, D. J. (2005). Neuropsychological intervention in kindergarten children with subtyped risks of reading retardation. Annals of Dyslexia, 55, 217-245.

Stuss, D. T., Picton, T. W., \& Cerri, A. M. (1986). Searching for the name of pictures: an event-related potential study. Psychophysiology, 23, 215-223.

Van der Schoot, M., Licht, R., Horsley, T. M., \& Sergeant, J. A. (2000). Inhibitory deficits in reading disability depend on subtype: guessers but not spellers. Child Neuropsychology, 6, 297-312.

Van der Schoot, M., Licht, R., Horsley, T. M., \& Sergeant, J. A. (2002). Fronto-central dysfunctions in reading disability depend on subtype: guessers but not spellers. Developmental Neuropsychology, 22, $533-564$

Van Strien, J. W. (1999). Verbal learning in boys with P-type dyslexia, L-type dyslexia, and boys without learning disabilities: differences in learning curves and in serial position curves. Child Neuropsychology, $5,145-153$.

Van Strien, J. W., Bakker, D. J., Bouma, A., \& Koops, W. (1990). Familial resemblance for cognitive abilities in families with P-type dyslexic, L-type dyslexic, or normal reading boys. Journal of Clinical and Experimental Neuropsychology, 12, 843-856. 